\title{
Rapid RNA Exchange in Aqueous Two-Phase System and Coacervate Droplets
}

\author{
Tony Z. Jia • Christian Hentrich • Jack W. Szostak
}

Received: 13 November 2013 / Accepted: 31 January 2014 /

Published online: 28 February 2014

(C) The Author(s) 2014. This article is published with open access at Springerlink.com

\begin{abstract}
Compartmentalization in a prebiotic setting is an important aspect of early cell formation and is crucial for the development of an artificial protocell system that effectively couples genotype and phenotype. Aqueous two-phase systems (ATPSs) and complex coacervates are phase separation phenomena that lead to the selective partitioning of biomolecules and have recently been proposed as membrane-free protocell models. We show in this study through fluorescence recovery after photobleaching (FRAP) microscopy that despite the ability of such systems to effectively concentrate RNA, there is a high rate of RNA exchange between phases in dextran/polyethylene glycol ATPS and ATP/poly-L-lysine coacervate droplets. In contrast to fatty acid vesicles, these systems would not allow effective segregation and consequent evolution of RNA, thus rendering these systems ineffective as model protocells.
\end{abstract}

Keywords Prebiotic chemistry · Phase separation - Compartmentalization - Aqueous two-phase systems $\cdot$ Coacervates $\cdot$ Origin of life

\section{Introduction}

The RNA world hypothesis provides a conceptual framework for the early development of life on earth in which RNA functions both as a molecule capable of propagating genetic information and as a catalyst. The capacity of RNA to transmit genetic information is exemplified by the RNA viruses, which can have genomes up to $30 \mathrm{~kb}$ in length consisting entirely of RNA (Lai and Cavanagh 1997). Ribozymes generated by in vitro directed RNA sequence evolution (Ellington and Szostak 1990; Tuerk and Gold 1990) and natural ribozymes such as selfsplicing introns (Cech et al. 1981; Kruger et al. 1982) are important examples of catalytic

Electronic supplementary material The online version of this article (doi:10.1007/s11084-014-9355-8) contains supplementary material, which is available to authorized users.

T. Z. Jia • C. Hentrich • J. W. Szostak $(\bowtie)$

Howard Hughes Medical Institute, Department of Molecular Biology, and Center for Computational and Integrative Biology, Massachusetts General Hospital, 185 Cambridge Street, Boston, MA 02114, USA e-mail: szostak@molbio.mgh.harvard.edu 
RNAs that serve as paradigms for the catalytic role of RNA in a prebiotic world. RNA molecules with RNA polymerase activity have been evolved in the laboratory (Johnston et al. 2001; Attwater et al. 2013), and a pair of RNA ligase ribozymes have been shown to crossreplicate each other by ligation in an exponential manner (Lincoln and Joyce 2009). Although RNA-catalyzed RNA replication is likely to have been important for primitive cells in the RNA world, it is also possible that non-enzymatic RNA replication may have played an important role in the transition from prebiotic chemistry to the emergence of the first cells. Since at this stage neither RNA-catalyzed nor purely chemically driven RNA replication have been demonstrated, it is reasonable to consider routes to the assembly of protocells based on either mode of RNA replication. In either case, replicating RNAs must be compartmentalized to allow for the evolution of functional RNAs that confer a selective advantage to the protocell within which they reside.

While there has been great progress in understanding prebiotically plausible vesicle assembly and replication pathways (Budin and Szostak 2010; Chen and Walde 2010), combining both encapsulation and replication into a functional model protocell presents additional challenges. Compartmentalization of genomic RNA molecules without (or with only rare) exchange between protocells is essential for any protocell model as it would allow RNA sequences with desirable properties, such as catalytic ribozymes, to be segregated from other RNAs and to selectively replicate and evolve over time (Szostak et al. 2001; Szabo et al. 2002). Phospholipids are the major building blocks in modern cell membranes, however phospholipid membranes are largely impermeable to charged molecules (Chen and Walde 2010) and are therefore problematic as the basis of protocell compartmentalization. However, membranes composed of fatty acids and related single chain amphiphiles are permeable to small polar and even charged molecules, and have additional properties that are favorable for protocell growth and division (Budin and Szostak 2011). Nevertheless, the simplicity of membrane free protocell models is intriguing and makes such systems worth further exploration.

Droplets formed by phase separation in an aqueous environment, such as aqueous twophase systems (ATPS) and charge-complex coacervates, have been proposed as model protocells (Oparin 1953; Fox 1976; Liebl et al. 1984; Koga et al. 2011; Keating 2012; Mann 2012, 2013). Both ATPSs (Albertsson 1971; Walter et al. 1985; Zaslavsky 1995) and coacervates (Dufrenoy and Reed 1946; Oparin et al. 1961) have long been known to lead to the partitioning of specific molecules into different phases in an overall aqueous environment. In biotechnological applications, ATPSs composed of dextran and polyethylene glycol (PEG) are commonly used to partition whole bacterial cells (Stendahl et al. 1977), cellular organelles (Albertsson 1958), and macromolecules (Hatti-kaul 2001); RNA, for example, partitions into the more polar dextran-rich phase (Zaslavsky 1992). Some properties of ATPSs and coacervates could have been advantageous in the development of early cells. Their ability to concentrate primitive reactants and catalysts, such as ribozymes, could increase reaction rates without requiring a lipid-based boundary (Strulson et al. 2012). Both ATPSs and coacervates also function as compartments in vitro (Williams et al. 2012; Strulson et al. 2012) and in the case of a dextran/PEG ATPS, within a phospholipid vesicle (Helfrich et al. 2002; Long et al. 2005). Coacervate droplets are particularly attractive due to the simplicity of their components, e.g. mononucleotides and small polypeptides, both of which could have been produced in a prebiotic environment (Leman et al. 2004; Powner et al. 2009).

Because of the membrane-independent nature of ATPS and coacervate models, it is unclear whether these systems are able to compartmentalize genetic molecules such as RNA with minimal exchange between droplets. We have therefore studied the ability of ATPS and coacervate droplets to retain RNA oligonucleotides 15 and 50 nucleotides in length, and thereby gauge their effectiveness as membrane-free protocell model systems. 


\section{Results}

Properties of ATPS and Coacervate Systems

A $16 \%$ dextran $/ 10 \%$ PEG (initial $w / v$ ) ATPS was prepared, yielding roughly equal volumes of the dextran-rich and PEG-rich phases (Fig. S1a). When the ATPS was mixed by vortexing, a turbid suspension consisting of small, dispersed dextran-rich droplets in the bulk PEG-rich phase and PEG-rich droplets in the bulk dextran-rich phase formed. After several minutes the droplets began to coalesce and the system separated into two clear phases (Fig. S1b), with the dextran-rich phase at the bottom due to its greater density. Whether the system was in a dispersed or a coalesced state, we observed a rapid 8-fold enrichment of a fluorescently labeled RNA 15-mer into the dextran-rich phase; the fluorescent dye did not have a strong effect on partitioning (Table S1).

We also investigated partitioning of RNA in ATPSs made using PEG and ionic derivatives of dextran, including cationic diethylaminoethyl dextran (DEAE-dextran) and anionic dextransulfate (Fig. S2). As expected, both of the PEG/dextran derivative systems lead to a greater degree of partitioning of RNA (Table S1). In a $25 \%$ DEAE-dextran/25\% PEG $(w / v)$ system (yielding $\approx 55 \%$ DEAE-dextran-rich phase by volume), RNA partitioned strongly into the DEAE-dextran-rich phase due to the positive charge of the DEAE-dextran and the more polar nature of that phase; the degree of partitioning was so great that the RNA concentration in the PEG-rich phase was below our detection limit (Table S1). Conversely, in a $16 \%$ dextransulfate/10 \% PEG $(w / v)$ system ( $\approx 60 \%$ dextran-sulfate-rich phase by volume), RNA partitioned strongly into the PEG-rich phase, presumably due to charge repulsion from the anionic dextran-sulfate. Droplets in the DEAE-dextran/PEG system coalesced more slowly than droplets in the dextran/PEG or dextran-sulfate/PEG system (Fig. S3), most likely due to the high viscosity of DEAE-dextran. In all systems, renewed vortexing or mixing led to the reformation of the turbid state consisting of small, dispersed droplets.

We also prepared coacervates consisting of complexes of anionic ATP and cationic poly-Llysine (pLys). Upon visual inspection, the ATP/pLys system (30 mM ATP, $2 \%$ pLys) appeared similar to the ATPSs as two phases formed under specific concentration conditions (Fig. S4a). Following coalescence, the lower, more dense phase was highly enriched in ATP/pLys complexes formed by the charge balancing of these species (Fig. S4b). In order for coacervates to form, a number of parameters must fall within defined limits, including the ATP and pLys concentrations (Koga et al. 2011), salt concentrations (Fig. S5), nucleotides (Table S2), and the molecular weight of the pLys (Table S2). RNA oligomers partitioned strongly into the complex-enriched phase to a degree that was comparable to that of the DEAE-dextran/PEG system (Table S1).

\section{RNA Retention in ATPS and Coacervate Droplets}

We sought to determine the ability of ATPS and coacervate droplets to retain RNA in a manner similar to fatty acid based vesicles by preparing droplets into which a fluorescently labeled RNA 15-mer oligonucleotide had partitioned. We then used fluorescence recovery after photobleaching (FRAP) microscopy to analyze the rates at which the RNA moved from the bulk phase into photo-bleached droplets. At steady state, this would be equivalent to the rate at which RNA diffused out of droplets into the bulk phase (and then into other droplets). We acquired and analyzed fluorescence recovery data for fluorescently labeled RNA in droplets from four systems (Table S3): $16 \%$ dextran/10 \% PEG (Fig. 1a, Movie S1), $25 \%$ DEAEdextran/25 \% PEG (Fig. 1b, Movie S2), 16 \% dextran-sulfate/10 \% PEG (Fig. 1c, Movie S3), 

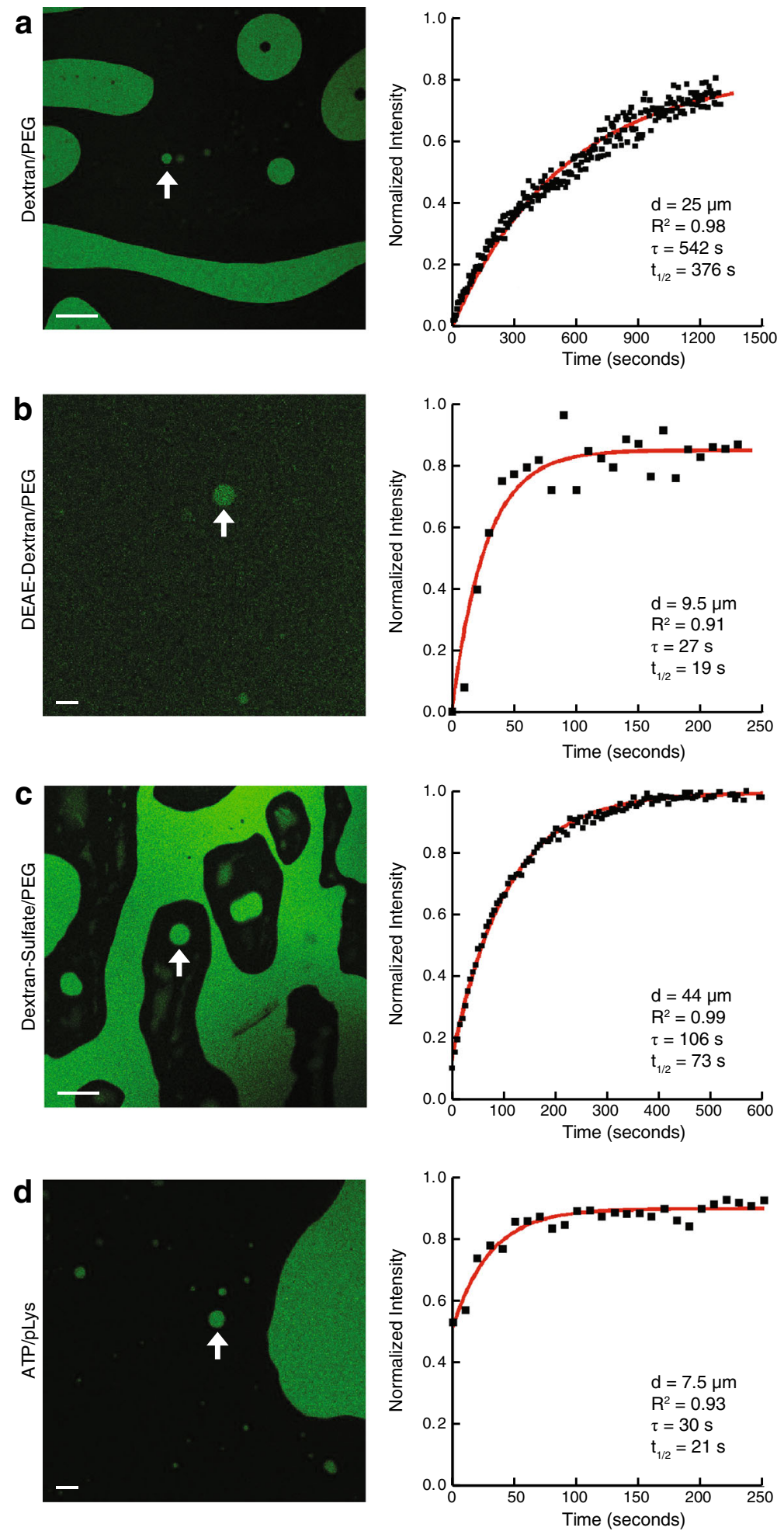
Fig. 1 Rapid exchange of RNA oligomers between ATPS and coacervate droplets and the surrounding bulk phase. Representative confocal fluorescence images showing RNA enriched droplets (green) are shown at left. Normalized fluorescence recovery after photobleaching (FRAP) recovery curves are shown at right. All samples contained $5 \mu \mathrm{M}$ 5'-6-FAM-labeled RNA 15-mer (5'-CCAGUCAGUCUACGC-3') in: (a) $16 \%$ dextran 9$11 \mathrm{kDa} / 10 \%$ PEG $8 \mathrm{kDa}$ in $50 \mathrm{mM}$ Tris-Cl pH 8 and $100 \mathrm{mM} \mathrm{NaCl}$ (indicated droplet $25 \mu \mathrm{m}$ diameter), (b) $25 \%$ DEAE-dextran $>500 \mathrm{kDa} / 25 \%$ PEG $8 \mathrm{kDa}$ in $100 \mathrm{mM}$ Tris-Cl pH 8 with the GODCAT (glucose oxidase/ catalase) system (Methods) (indicated droplet $9.5 \mu \mathrm{m}$ diameter), (c) $16 \%$ dextran-sulfate $9-20 \mathrm{kDa} / 10 \% \mathrm{PEG}$ $8 \mathrm{kDa}$ in $50 \mathrm{mM}$ Tris-Cl pH 8 and $100 \mathrm{mM} \mathrm{NaCl}$ (indicated droplet $44 \mu \mathrm{m}$ diameter), (d) $30 \mathrm{mM} \mathrm{ATP} / 2 \% \mathrm{pLys}$ 4-15 kDa in $100 \mathrm{mM}$ Tris-Cl pH 8 with the GODCAT system (Methods) (indicated droplet $7.5 \mu \mathrm{m}$ diameter). See Movies S1-S4 for respective FRAP movies. Each curve was normalized to the intensities of a non-bleached droplet and the background within the same frame, to correct for photobleaching during sampling, as well as to its initial intensity, to account for variable photobleaching before the recovery step across runs (Supplementary Information). Data were fit to a single exponential to determine time constants $(\tau)$ and half-lives $\left(t_{1 / 2}\right)$ for fluorescence recovery (Supplementary Information). Further details and data in Table S3. Scale bars for (a) and (c) are $100 \mu \mathrm{m}$; scale bars for (b) and (d) are $10 \mu \mathrm{m}$. See Movies S1-S4 for full movies of photobleaching and recovery for each of the indicated droplets in (a)-(d), respectively. All percentages are $w / v$

and $30 \mathrm{mM} \mathrm{ATP} / 2 \%$ pLys (Fig. 1d, Movie S4) (all percentages $w / v$ ). The sizes of droplets ranged from 1 to $5 \mu \mathrm{m}$ in diameter (Fig. S6), similar in size to proposed fatty acid vesicle based protocell model systems (Adamala and Szostak 2013a), up to 50-75 $\mu \mathrm{m}$ in diameter (Fig. 1c), similar in size to giant unilamellar vesicles (Dimova et al. 2006).

In dextran-rich and DEAE-dextran-rich droplets (in their respective ATPSs) between $5 \mu \mathrm{m}$ and $10 \mu \mathrm{m}$ in diameter, the fluorescence recovery half-life $\left(\mathrm{t}_{1 / 2}\right)$ of the fluorescently labeled RNA oligonucleotides was 8-20 s (Table S3). In the dextran/PEG system, larger dextran-rich droplets $(20 \mu \mathrm{m}$ and $25 \mu \mathrm{m}$ in diameter) (Fig. S7) recovered fluorescence significantly more slowly than the other dextran-rich droplets measured, possibly due to their larger size and/or their greater distance from other droplets. The fluorescence of RNA-enriched PEG-rich droplets in the dextran-sulfate/PEG ATPS, despite being the largest droplets sampled in all systems, recovered more quickly than large droplets in the dextran/PEG system (Table S3).

The RNA-enriched ATP/pLys droplets also recovered fluorescence quickly after photobleaching. The rate of exchange of RNA between droplets and their surrounding bulk phase was similar to that seen in dextran and DEAE-dextran droplets of comparable size (Table S3). After photobleaching, the fluorescence recovery $\mathrm{t}_{1 / 2}$ was 5-21 s for the ATP/pLys droplets measured (3-9 $\mu \mathrm{m}$ in diameter) (Table S3).

To test the influence of length on RNA retention within droplets, we measured the fluorescence recovery $t_{1 / 2}$ after photobleaching of droplets of the dextran/PEG ATPS and the ATP/pLys system containing a fluorescently labeled RNA 50-mer. For the droplets measured in both of these systems, the fluorescence recovery $\mathrm{t}_{1 / 2}$ was $11-76 \mathrm{~s}(4-11 \mu \mathrm{m}$ in diameter) (Table S4). Compared to similar-sized droplets in their respective systems containing the RNA 15-mer (Table S3), droplets containing the longer RNA resulted in a modest increase of the fluorescence recovery $t_{1 / 2}$ by a factor of roughly 3 .

To compare the time scale of RNA retention between phase-separated droplet systems and fatty acid vesicles, we prepared oleic acid vesicles, similar in size to the droplets studied above, that contained the fluorescently labeled RNA 15-mer. For the vesicle experiments, a high concentration of fluorescently labeled RNA was present outside of the vesicles as well. Ten minutes after photobleaching a sample, the external solution had fully recovered in fluorescence intensity due to the diffusion of RNA from adjacent non-bleached sample regions. However, the vesicles did not regain any detectable internal fluorescence intensity (Fig. 2, Movie S5). As expected, fatty acid vesicles, despite being more permeable to charged species than phospholipid vesicles, did not exhibit measurable permeability for RNA oligomers. The rate of RNA exchange across a fatty acid vesicle membrane was several orders of magnitude slower than the rate of RNA exchange across the boundaries of ATPS or coacervate droplets. 

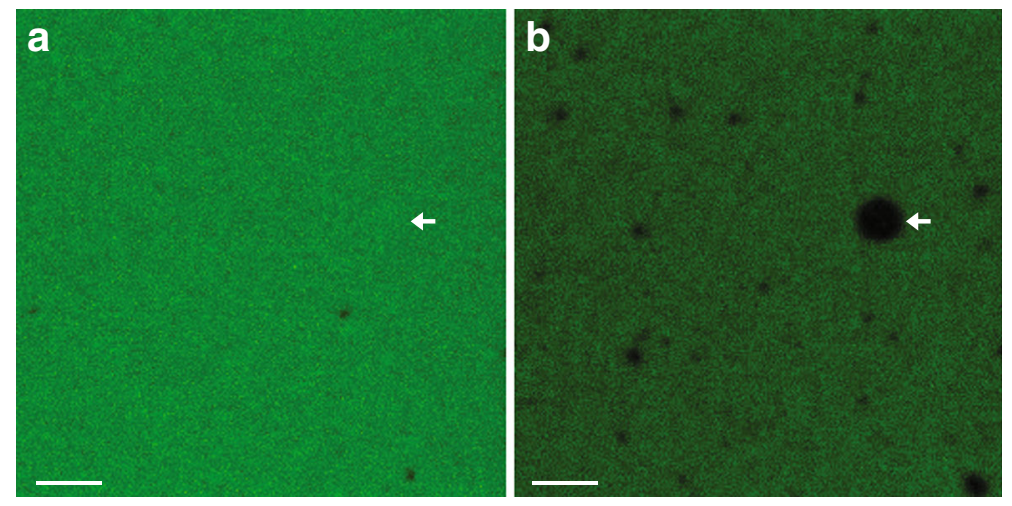

Fig. 2 Oleic acid vesicles do not exchange RNA with the surrounding fluid. Representative confocal microscope images of a sample (a) before photobleaching and (b) $590 \mathrm{~s}$ after photobleaching of the indicated non-gel-filtered oleic acid vesicle in $200 \mathrm{mM}$ Bicine- $\mathrm{NaOH}$ pH 8.5 containing 5'-6-FAM labeled RNA 15-mer (5'-CCAGUCAG UCUACGC-3') at room temperature (Methods). The vesicle samples were not gel filtered in order to maintain a high RNA concentration outside of the vesicles in order to simulate conditions similar to the ATPS and coacervate systems. After the entire window was photobleached, fluorescence outside of the vesicles recovered due to rapid RNA diffusion, but fluorescence inside vesicles did not recover due to lack of transport of RNA across the membrane. Scale bars, $10 \mu \mathrm{m}$. See Movie S5 for full movie of photobleaching and recovery

This observation clearly demonstrates that although dextran/PEG ATPS and ATP/pLys coacervate systems are able to concentrate RNA molecules through partitioning into droplets, their ability to compartmentalize these oligomers over time scales longer than seconds was insignificant compared to that of fatty acid vesicles.

We then asked whether combining a dextran/PEG ATPS or an ATP/pLys coacervate system with current vesicle systems would allow RNA partitioning within a model protocell. Previous work has shown that it is possible to form phospholipid vesicles that contain dextran/PEG ATPSs (Helfrich et al. 2002; Long et al. 2005; Dominak et al. 2010), and that these systems are able to partition RNA to sub-regions within a vesicle. We were able to encapsulate a dextran/ PEG ATPS inside oleic acid vesicles (Fig. 3). As expected, the fluorescently labeled RNA 15mer partitioned into the dextran-rich phase inside oleate vesicles, providing an RNA-rich compartment within these vesicles. However, the ATP/pLys system used in this study was not compatible with fatty acids. Attempts to produce fatty acid vesicles containing the ATP/pLys system resulted in quantitative precipitation of the fatty acids, most likely due to the charge interactions between the cationic lysine side chain and anionic fatty acid molecules.

\section{Discussion}

For the ATPS and coacervate droplets studied, exchange of RNA across the droplet boundary occurred orders of magnitude more rapidly than across the membrane of fatty acid vesicles. Although our FRAP measurements report only on the entry of RNA oligomers into ATPS or coacervate droplets, at steady state, the rate of efflux of RNA from droplets must equal the rate of influx. Our data therefore imply that RNA molecules do not remain localized within any droplet for longer than a period of seconds, and rapidly exchange between droplets via the surrounding bulk phase. Although a larger RNA such as a ribozyme would diffuse more slowly in solution due to its greater mass, our data indicates that longer RNAs will not reside in a droplet for a significantly longer time before diffusing out of the droplet. Fast RNA exchange 


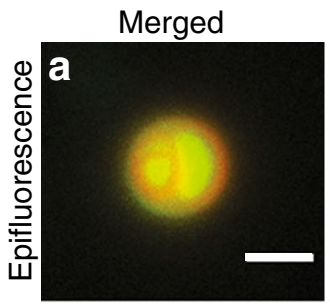

Merged

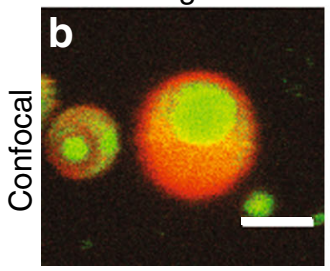

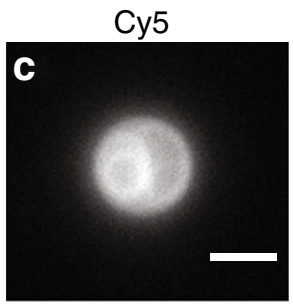

Cy5

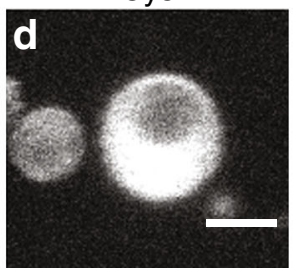

HPTS

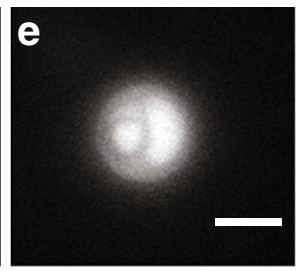

HPTS

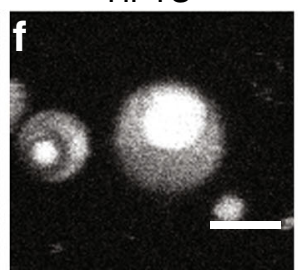

Phase Contrast

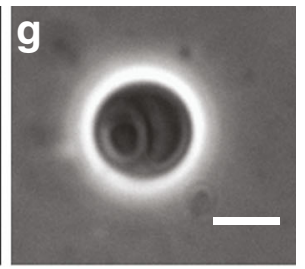

Bright Field

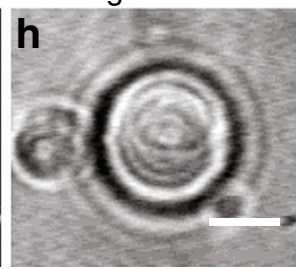

Fig. 3 Formation of a dextran-PEG ATPS inside oleate vesicles. (a) and (b): Merged images of Cy5-RNA fluorescence (red, Dextran-rich phase) and 8-hydroxypyrene-1,3,6-trisulfonate (HPTS) fluorescence (green, PEG-rich phase). (c) and (d): the individual Cy5-RNA fluorescence channels for (a) and (b), respectively. (e) and (f): the HPTS fluorescence channels for (a) and (b), respectively. (g) and (h): Corresponding phase contrast (top) and bright field images (bottom). Images in the top row were acquired sequentially using an epifluorescence microscope; images in the bottom row were acquired simultaneously using confocal microscopy. Cy5-labeled RNA partitioned strongly into the dextran-rich phase, and HPTS partitioned into the PEG-rich phase. The dextran-rich (red) and the PEG-rich (green) phases could separate spontaneously within an oleic acid vesicle. See Methods for details. Scale bars, $3 \mu \mathrm{m}$

coupled with the observed rapid coalescence of droplets suggests that ATPS and coacervate droplets would not confer the stable compartmentalization necessary for multiple generations of RNA selection and replication to occur, which would need to be on the order of many hours, if not days (Deck et al. 2011; Adamala and Szostak 2013b). If a given RNA molecule only resides in a particular droplet for a short period of time before exchanging into a different droplet, the products of any functional activity of that RNA (such as the catalytic production of a useful metabolite) would be spread across many droplets, and furthermore would not be heritable. In essence, the rapid exchange of RNA molecules between droplets is equivalent to a lack of compartmentalization in a time-averaged sense. Darwinian evolution requires compartmentalization so that mutations that improve function can lead to a selective advantage for the mutant genomic molecule. As the capacity for Darwinian evolution is a basic requirement for any protocell model, it is clear that unmodified ATPS and coacervate droplets are unsuitable protocell models.

To decrease the rate of RNA exchange between droplets, it may be productive to consider systems in which RNA molecules could covalently attach to a matrix or to particles that would stay localized within a droplet. Many RNA affinity purification techniques rely on covalent attachments to a matrix such as sepharose (Allerson et al. 2003) or agarose beads (Caputi et al. 1999) and such a system could serve to slow RNA exchange. The coacervate system we studied was composed of a simple polypeptide (pLys) and a simple mononucleotide (ATP). RNA-protein (Lee et al. 1977; Drygin 1998; Baskerville and Bartel 2002) or RNA-nucleotide (Flügel and Wells 1972; Flügel et al. 1973) covalent interactions produced by photocrosslinking could be good starting points to develop a system in which RNA becomes covalently linked to a matrix within coacervate droplets in a prebiotically plausible manner. Alternatively, utilizing a poly(Arg) matrix could result in tightly bound RNA that is unable to diffuse away from the droplet due to the electrostatic interactions between the arginine 
side-chains and the RNA backbone (Knight and Landweber 1998). Other immobilization techniques that take advantage of the ability of RNA to form base-pairs could also serve to slow RNA exchange.

Although dextran/PEG ATPS and ATP/pLys coacervate systems do not provide suitably stable compartmentalization of reactants for long periods of time, such systems do enable transient localization and concentration of RNA molecules. Focusing on the potential usefulness of these systems for sub-compartmentalization within protocells may be a productive direction for future research (Hyman and Brangwynne 2011). Fatty acid and phospholipid vesicle systems compatible with dextran/PEG ATPSs have been developed (Helfrich et al. 2002; Long et al. 2005; Dominak et al. 2010; this study), and it may be possible to develop similar vesicle systems that are compatible with the ATP/pLys coacervate system. This might be achieved by using net-neutral zwitterionic phospholipids or non-ionic amphiphiles as membrane forming molecules, as they would not interact strongly with the coacervate components, thus avoiding precipitation. Such a system would be similar to cellular organelle-based compartmentalization. In a prebiotic setting, a lipid-based membrane could encapsulate all components, and selective chemical partitioning into the two phases could provide an early protocell with the ability to partition compounds internally and accelerate reactions within the protocell, including for example the assembly of RNA complexes and ribozyme catalysis (Strulson et al. 2012). Thus, understanding how ATPSs and coacervates interact and combine with fatty acid and phospholipid vesicles may lead to a greater understanding of the possibilities for the development of early cells in an RNA world.

\section{Methods}

Chemicals Tris(hydroxymethyl) aminomethane (Tris), sodium chloride, magnesium chloride hexahydrate, D-(+)-glucose, 2-mercaptoethanol, adenosine 5'-triphosphate (ATP) disodium salt hydrate, adenosine 5'-diphosphate (ADP) disodium salt, adenosine 5'-monophosphate (AMP) disodium salt, guanosine 5'-triphosphate (GTP) sodium salt hydrate, guanosine 5'diphosphate (GDP) sodium salt, guanosine 5'-monophosphate (GMP) disodium salt hydrate, uridine 5'-triphosphate (UTP) trisodium salt hydrate, 8-hydroxypyrene-1,3,6-trisulfonic acid (HPTS) trisodium salt, enzyme catalase from bovine liver, polyethylene glycol (PEG) $8 \mathrm{kDa}$, dextran 9-11 kDa from Leuconostoc mesenteroides, dextran sulfate sodium salt 9-20 kDa from Leuconostoc spp., diethylaminoethyl-dextran (DEAE-dextran) hydrochloride $>500 \mathrm{kDa}$, poly-L-lysine (pLys) hydrobromide 1-5 kDa, poly-L-lysine hydrobromide 4-15 kDa, poly-Llysine hydrobromide 15-30 kDa, and Sepharose 4B (45-165 $\mu \mathrm{m}$ bead diameter) beads were purchased from Sigma-Aldrich Corporation (St. Louis, MO).

RNA oligonucleotides [(5'-CCAGUCAGUCUACGC-3') both unlabeled and 6carboxyfluorescein (6-FAM) 5'-labeled, 6-FAM 5'-labeled (5'- CAUCUAGUUACCUCUA GGAUCUCAUGAUGCCUGAAGCGUAGACUGACUGG-3'), and 5'-Cy5-labeled (5'GCGUAGACUGACUGG-3')] were purchased from Integrated DNA Technologies (Coralville, IA) and used without further purification. dNTPs and cytidine 5'-triphosphate (CTP) sodium salt were purchased from GE Healthcare (Little Chalfont, United Kingdom). Oleic acid was purchased from Nu-Chek Prep, Inc. (Elysian, MN). rNTPs and glass microscope slides $(25 \mathrm{~mm} \times 75 \mathrm{~mm}, 1 \mathrm{~mm}$ thick) were purchased from VWR International (Radnor, PA). Glucose oxidase from Aspergillus was purchased from Serva Electrophoresis (Heidelberg, Germany). Glass cover slips $(18 \times 18 \mathrm{~mm}$ No. 1) were purchased from Thermo Fisher Scientific (Waltham, MA). All solutions were produced in nuclease-free water from BioExpress (Kaysville, UT). 
Preparation of ATPS and Coacervate Samples A $16 \% w / v$ dextran 9-11 kDa and $10 \% w / v$ PEG $8 \mathrm{kDa}$ solution was prepared by dissolving the solid components in a solution of $50 \mathrm{mM}$ Tris-Cl pH 8 and $100 \mathrm{mM} \mathrm{NaCl}$ (Strulson et al. 2012) with vigorous vortexing for a few minutes. The $16 \% w / v$ dextran-sulfate sodium salt 9-20 kDa and $10 \% w / v$ PEG $8 \mathrm{kDa}$ was prepared by dissolving the solid components in a solution of $50 \mathrm{mM}$ Tris-Cl $\mathrm{pH} 8$ and $100 \mathrm{mM}$ $\mathrm{NaCl}$ with moderate vortexing for several seconds. The $25 \% \mathrm{w} / v$ DEAE-dextran hydrochloride $>500 \mathrm{kDa}$ and $25 \% \mathrm{w} / v$ PEG $8 \mathrm{kDa}$ was prepared by dissolving the solid components in a solution of $100 \mathrm{mM}$ Tris- $\mathrm{Cl} \mathrm{pH} 8$ with vigorous vortexing and heating to $65{ }^{\circ} \mathrm{C}$ for several minutes. $30 \mathrm{mM}$ ATP - 2\% $\% / v$ pLys (either $1-5 \mathrm{kDa}, 4-15 \mathrm{kDa}$, or $15-30 \mathrm{kDa}$ as indicated) was prepared by mixing respective stock solutions (200 mM ATP and $10 \%$ or $50 \% w / v$ pLys both in $100 \mathrm{mM}$ Tris-Cl pH 8) and diluting with $100 \mathrm{mM}$ Tris-Cl pH 8. All samples were prepared in $1.5 \mathrm{~mL}$ eppendorf tubes at room temperature. Due to the viscosity of the DEAEdextran/PEG sample, pipet tips that were cut roughly $1 \mathrm{~cm}$ from the tip were used for that sample. To each sample, 5'-6-FAM-labeled RNA (5'- CCAGUCAGUCUACGC-3' or 5'CAUCUAGUUACCUCUAGGAUCUCAUGAUGCCUGAAGCGUAGACUGACUGG-3') from a $100 \mu \mathrm{M}$ stock solution in nuclease-free water was added to a final concentration of $5 \mu \mathrm{M}$ RNA. Each solution was vortexed for $30 \mathrm{~s}$. For applications that required the two phases to be separated, the sample tube was centrifuged for $15 \mathrm{~min}$ at 14,000 rpm. Each phase was then pipetted into separate tubes. Transmittance measurements were performed using a GE Healthcare (formerly Amersham) Ultrospec 3,100 pro UV-Visible spectrometer (Little Chalfont, United Kingdom). RNA phase-specific partitioning measurements were performed using a Thermo Fisher Scientific (Waltham, MA) Nanodrop 2000c Spectrophotometer. For confocal microscopy, DEAE-dextran/PEG and ATP/pLys samples also contained the GODCAT system (Glucose Oxidase-Catalase) to reduce photobleaching (Hentrich and Surrey 2010), and included $2 \% \mathrm{w} / v \mathrm{D}-(+)$-glucose, $0.5 \mathrm{mg} / \mathrm{mL}$ catalase, $1 \mathrm{mg} / \mathrm{mL}$ glucose oxidase, and $143 \mathrm{mM} 2$-mercaptoethanol. A $6-8 \mu \mathrm{L}$ droplet $(6 \mu \mathrm{L}$ for dextran/PEG, dextransulfate/PEG samples, and ATP/pLys samples, and $8 \mu \mathrm{L}$ for DEAE-dextran/PEG samples) for each sample was applied to each glass slide $(25 \times 75 \mathrm{~mm})$ and a cover slip (18x18mm, No. 1$)$ was applied. The slide was allowed to sit at room temperature until the droplet applied was completely spread across the entire cover slip area, and then the cover slip was sealed using Valap (1:1:1 vaseline, lanolin, paraffin wax) to avoid evaporation. Samples were covered with aluminum foil to reduce photobleaching by stray light until imaging.

Preparation of Oleic Acid Vesicle Samples $\sim 10 \mathrm{mM}$ oleic acid vesicles containing 5'-6-FAMlabeled RNA (5'-CCAGUCAGUCUACGC-3') were prepared by mixing $1.6 \mu \mathrm{L}$ pure oleic acid $(3.17 \mathrm{M})$ with $50 \mu \mathrm{L}$ of $10 \mu \mathrm{M}$ RNA in $500 \mu \mathrm{L} 180 \mathrm{mM}$ bicine buffer adjusted to $\mathrm{pH} 8.5$ with $\mathrm{NaOH}$, followed by vortexing for $30 \mathrm{~s}$. The sample was covered with foil and allowed to gently tumble overnight. A $3 \mu \mathrm{L}$ droplet was applied to a glass slide as above for microscopy. The glass slide was then allowed to sit (cover slip down) at room temperature for $30 \mathrm{~min}$ to allow larger vesicles to rest on the surface of the cover slip.

Preparation of a Dextran/PEG ATPS Inside Oleic Acid Vesicles To $840 \mu \mathrm{L}$ of $5.95 \%$ PEG $8 \mathrm{kDa}, 10.7 \%$ Dextran $10 \mathrm{kDa}, 200 \mathrm{mM}$ bicine $\mathrm{pH} 8.5$ (adjusted with $\mathrm{NaOH}$ ), $0.5 \mu \mathrm{L} 200 \mathrm{mM}$ HPTS (8-hydroxypyrene-1,3,6-trisulfonate, stock in $\mathrm{H}_{2} \mathrm{O}, 0.12 \mathrm{mM}$ final concentration) and $10 \mu \mathrm{L}$ of $100 \mu \mathrm{M}$ 5'-Cy5-labeled RNA (5'-GCGUAGACUGACUGG-3' in $\mathrm{H}_{2} \mathrm{O}, 1.2 \mu \mathrm{M}$ final concentration) were added. The solution was vigorously vortexed and visually inspected to verify that it contained only one phase. Subsequently, $3 \mu \mathrm{L}$ of oleic acid were added to the solution and after another vigorous vortexing, the solution was tumbled over night on a rotating wheel $(6 \mathrm{rpm})$ to allow vesicle formation. The next day, the vesicles were purified 
from unencapsulated dye and RNA using a short $1 \mathrm{~cm}$ Sepharose 4B gel filtration column and $1 \mathrm{mM}$ oleic acid in $200 \mathrm{mM}$ bicine (adjusted to $\mathrm{pH} 8.5$ with $\mathrm{NaOH}$ ) as a running buffer. $6 \mu \mathrm{L}$ of gel-filtered vesicles were spread out (to around $1 \mathrm{~cm}^{2}$ ) on a $25 \times 75 \mathrm{~mm}$ microscope slide and the droplet was allowed to evaporate for $6 \mathrm{~min}$ at room temperature. Then an $18 \times 18 \mathrm{~mm}$ coverslip was placed onto the droplet and the slide was sealed using Valap. Alternatively, a $3 \mu \mathrm{L}$ droplet was placed on a slide and a coverslip was placed immediately on top of it. In this case, the coverslip was not sealed, but only fixed in the corners with Valap, and evaporation was allowed to occur through the edges over several hours. Slides were observed either with a confocal microscope (see below) or with a Nikon (Tokyo, Japan) TE2000 inverted fluorescence microscope with a $100 \times$ oil objective.

Fluorescence Recovery After Photobleaching (FRAP) by Confocal Microscopy Each sample was imaged using a confocal microscope at $488 \mathrm{~nm}$ (pinhole $1 \mathrm{AU}$ ). Confocal microscopy was performed using a Leica (Solms, Germany) SP5 AOBS Scanning Laser Confocal Microscope (63×, 1.4-0.6 N. A. Plan-Apochromat oil immersion objective or $20 \times, 0.7$ N. A. air objective), a Carl Zeiss (Oberkochen, Germany) LSM 510 Laser Scanning Microscope (63×, 1.4 N. A. PlanApochromat oil immersion objective), or a Nikon (Tokyo, Japan) A1R Confocal Microscope $(60 \times, 1.49$ N. A. Apochromat TIRF oil immersion objective). After selection of the droplet to be analyzed, a time zero image was acquired, and then a circular or square region was photobleached at high power using an Argon laser at $488 \mathrm{~nm}$ (or a solid state laser for the Nikon system). Each photobleaching region was chosen to be as small as possible while still containing a single, whole droplet to minimize collateral photobleaching of neighboring droplets. The fluorescence intensity (either $493 \mathrm{~nm}$ to $543 \mathrm{~nm}$ on the Leica system, $505 \mathrm{~nm}$ to $530 \mathrm{~nm}$ on the Zeiss system, or $500 \mathrm{~nm}$ to $550 \mathrm{~nm}$ on the Nikon system) was then measured over time to track the fluorescence recovery of 5'-6-FAM-labeled RNA molecules within the droplet of interest.

Image and Data Analysis Curve fitting of the fluorescence recovery after photobleaching (FRAP) intensities was carried out by first obtaining intensities across all time points of a specific droplet. These intensities were normalized to the intensities of a non-bleached droplet and the background within the same frame, to correct for nonspecific photobleaching during sampling. The intensities were then normalized to the initial intensity of the droplet analyzed, to account for variable photobleaching before the recovery step across runs (Phair et al. 2004). Curves were then fit to a single exponential recovery function. See Supplemental Information for detailed explanation of image analysis and curve fitting. All imaging visualization, analysis, calculations, and production of movies were performed using FIJI (Fiji is Just ImageJ). All curve fitting was performed using MATLAB (Natick, MA). All figures were produced using Adobe Illustrator (San Jose, CA).

Open Access This article is distributed under the terms of the Creative Commons Attribution License which permits any use, distribution, and reproduction in any medium, provided the original author(s) and the source are credited.

\section{References}

Adamala K, Szostak JW (2013a) Competition between model protocells driven by an encapsulated catalyst. Nat Chem 5:495-501 
Adamala K, Szostak JW (2013b) Nonenzymatic template-directed RNA synthesis inside model protocells. Science 342:1098-1100

Albertsson P-A (1958) Particle fractionation in liquid two-phase systems: the composition of some phase systems and the behaviour of some model particles in them application to the isolation of cell walls from microorganisms. Biochim Biophys Acta 27:378-395

Albertsson P-A (1971) Partition of cell particles and macromolecules. Wiley-Interscience, New York

Allerson CR, Martinez A, Yikilmaz E, Rouault TA (2003) A high-capacity RNA affinity column for the purification of human IRP1 and IRP2 overexpressed in Pichia pastoris. RNA 9:364-374

Attwater J, Wochner A, Holliger P (2013) In-ice evolution of RNA polymerase ribozyme activity. Nat Chem 5:1-8

Baskerville S, Bartel DP (2002) A ribozyme that ligates RNA to protein. Proc Natl Acad Sci U S A 99:9154-9159

Budin I, Szostak JW (2010) Expanding roles for diverse physical phenomena during the origin of life. Annu Rev Biophys 39:245-263

Budin I, Szostak JW (2011) Physical effects underlying the transition from primitive to modern cell membranes. Proc Natl Acad Sci U S A 108:5249-5254

Caputi M, Mayeda A, Krainer AR, Zahler AM (1999) HnRNP A/B proteins are required for inhibition of HIV-1 pre-mRNA splicing. EMBO J 18:4060-4067

Cech TR, Zaug AJ, Grabowski PJ (1981) In vitro splicing of the ribosomal RNA precursor of tetrahymena: involvement of a guanosine nucleotide in the excision of the intervening sequence. Cell 27:487-496

Chen IA, Walde P (2010) From self-assembled vesicles to protocells. Cold Spring Harb Persp Biol 2:a002170

Deck C, Jauker M, Richert C (2011) Efficient enzyme-free copying of all four nucleobases templated by immobilized RNA. Nat Chem 3:603-608

Dimova R, Aranda S, Bezlyepkina N et al (2006) A practical guide to giant vesicles. Probing the membrane nanoregime via optical microscopy. J Phys Condens Matter 18:S1151-S1176

Dominak LM, Gundermann EL, Keating CD (2010) Microcompartmentation in artificial cells: pH-induced conformational changes alter protein localization. Langmuir 26:5697-5705

Drygin YF (1998) Natural covalent complexes of nucleic acids and proteins: some comments on practice and theory on the path from well-known complexes to new ones. Nucl Acids Res 26:4791-4796

Dufrenoy J, Reed HS (1946) The respiratory processes in plant cells in relation to the formation of coacervates. Plant Physiol 4:941-946

Ellington AD, Szostak JW (1990) In vitro selection of RNA molecules that bind specific ligands. Nature 346: $818-822$

Flügel RM, Wells RD (1972) Nucleotides at the RNA-DNA covalent bonds formed in the endogenous reaction by the avian myeloblastosis virus DNA polymerase. Virology 48:394-401

Flügel RM, Rapp U, Wells RD (1973) RNA-DNA covalent bonds between the RNA primers and the DNA products formed by RNA tumor virus DNA polymerase. J Virol 12:1491-1502

Fox SW (1976) The evolutionary significance of phase-separated microsystems. Orig Life Evol Biosph 7:49-68

Hatti-kaul R (2001) Aqueous two-phase systems. Mol Biotechnol 19:697-713

Helfrich MR, Mangeney-Slavin LK, Long MS et al (2002) Aqueous phase separation in giant vesicles. J Am Chem Soc 124:13374-13375

Hentrich C, Surrey T (2010) Microtubule organization by the antagonistic mitotic motors kinesin-5 and kinesin14. J Cell Biol 189:465-480

Hyman AA, Brangwynne CP (2011) Beyond stereospecificity: liquids and mesoscale organization of cytoplasm. Devel Cell 21:14-16

Johnston WK, Unrau PJ, Lawrence MS et al (2001) RNA-catalyzed RNA polymerization: accurate and general RNA-templated primer extension. Science 292:1319-1325

Keating CD (2012) Aqueous phase separation as a possible route to compartmentalization of biological molecules. Acc Chem Res 45:2114-2124

Knight RD, Landweber LF (1998) Rhyme or reason: RNA-arginine interactions and the genetic code. Chem Biol 5:R215-R220

Koga S, Williams DS, Perriman AW, Mann S (2011) Peptide-nucleotide microdroplets as a step towards a membrane-free protocell model. Nat Chem 3:720-724

Kruger K, Grabowski PJ, Zaug AJ et al (1982) Self-splicing RNA: autoexcision and autocyclization of the ribosomal RNA intervening sequence of tetrahymena. Cell 31:147-157

Lai MMC, Cavanagh D (1997) The molecular biology of coronaviruses. Adv Virus Res 48:1-100

Lee YF, Nomoto A, Detjen BM, Wimmer E (1977) A protein covalently linked to poliovirus genome RNA. Proc Natl Acad Sci U S A 74:59-63

Leman L, Orgel L, Ghadiri MR (2004) Carbonyl sulfide-mediated prebiotic formation of peptides. Science 306 : 283-286

Liebl V, Novák VJA, Masinovský Z et al (1984) The evolution of prebiological self-organization: probable colloid-chemical evolution of first prokaryotic cells. Orig Life 14:323-334 
Lincoln TA, Joyce GF (2009) Self-sustained replication of an RNA enzyme. Science 323:1229-1232

Long MS, Jones CD, Helfrich MR et al (2005) Dynamic microcompartmentation in synthetic cells. Proc Natl Acad Sci U S A 102:5920-5925

Mann S (2012) Systems of Creation: The Emergence of Life from Nonliving Matter. Acc Chem Res 45:2131-2141

Mann S (2013) The origins of life: old problems, new chemistries. Angew Chem 52:155-162

Oparin AI (1953) The origin of life, 2nd edn. Dover Publishing, New York

Oparin AI, Serebrovskaia KB, Vasileva NV, Balaevsk TO (1961) Formation of coacervates from polypeptides+ polynucleotides. Dokl Akad Nauk 154:407-412

Phair RD, Gorski SA, Misteli T (2004) Measurement of dynamic protein binding to chromatin in vivo, using photobleaching microscopy. Method Enzym 375:393-414

Powner MW, Gerland B, Sutherland JD (2009) Synthesis of activated pyrimidine ribonucleotides in prebiotically plausible conditions. Nature 7244:239-242

Stendahl O, Edebo L, Magnusson KE et al (1977) Surface-charge characteristics of smooth and rough salmonella typhimurium bacteria determined by aqueous two-phase partitioning and free zones electrophoresis. Acta Pathol Microbiol Scand B: Microbiol Immunol 85B:334-340

Strulson CA, Molden RC, Keating CD, Bevilacqua PC (2012) RNA catalysis through compartmentalization. Nat Chem 4:941-946

Szabo P, Scheuring I, Czaran T, Szathmary E (2002) In silico simulations reveal that replicators with limited dispersal evolve towards higher efficiency and fidelity. Nature 420:340-343

Szostak JW, Bartel DP, Luisi PL (2001) Synthesizing life. Nature 409:387-390

Tuerk C, Gold L (1990) Systematic evolution of ligands by exponential enrichment: RNA ligands to bacteriophage T4 DNA polymerase. Science 249:505-510

Walter H, Brooks DE, Fisher D (1985) Partitioning in aqueous Two-phase systems: theory, methods, uses, and applications to biotechnology. Academic, New York

Williams DS, Koga S, Hak CRC et al (2012) Polymer/nucleotide droplets as bio-inspired functional microcompartments. Soft Matt 8:6004-6014

Zaslavsky BY (1992) Bioanalytical applications of partitioning in aqueous polymer two-phase systems. Anal Chem 64:765A-773A

Zaslavsky BY (1995) Aqueous two-phase partitioning: physical chemistry and bioanalytical applications. Marcel Dekker, New York 